\title{
Detecting complications in a patient with an epidural
}

E pidural analgesia is a popular technique for the management of postoperative pain after major surgery. They are often inserted in the anaesthetic room and managed initially in the recovery room. Recovery and anaesthetic nurses are often essential members of the team responsible for the safe and effective management of these patients.

There is no doubt that, in terms of efficay after major abdominal or thoracic surgery, epidural analgesia is superior to any other technique. An excellent review article has recently summarised the reported efficacy and complications of epidural analgesia after surgery (Wheatley et al, British Journal of Anaesthesia 2001: 87:47-61). With careful postoperative management, the excellent pain relief afforded by epidurals can be translated into improved patient outcome especially after major surgery and in high-risk patients. However, they have now been in use for several years and there may be a tendency to consider them as routine and risk-free. However, there are several potentially serious complications and you can play a vital role in reducing the incidence and outcome of these complications.

Patients with epidurals often spend considerable time in recovery before going back to the ward and it is during this period that several complications can arise. Perhaps the most common complication in the recovery room is hypotension. This is especially likely if the epidural has been inserted in a dehydrated patient undergoing emergency surgery. Consultant anaesthetists are constantly reminding trainees of the potential dangers of inserting epidurals in these patients, especially if dehydration and electrolyte disturbance has not been corrected completely. It is important to realise that, in patients with an epidural, even small amounts of blood loss or a slight deficit in fluid balance can lead to severe life-threatening hypotension. The incidence of significant hypotension in elective cases is reported in clinical studies as $0.7-3 \%$ but it likely to be much greater. A rapid reaction from you when hypotension occurs is essential.

Sometimes the epidural catheter is placed accidentally in the subarachnoid space (up to $1.2 \%$ ) or it may migrate there (up to $0.18 \%$ ). Under these circumstances, a normal epidural top-up dose (often given in recovery) will produce severe hypotension, motor block and respiratory distress. It is for this reason that it is wise to give a small test dose first. This is also essential to detect the very rare case of migration of the catheter into a vein.

Respiratory depression can occur in any patient if, as is usual practice with epidural analgesia, opioids are used. However, no special awareness is required for the recovery nurse who is, by definition, an expert in its detection and management.

An epidural haematoma often causes profound or total sensory and motor block which becomes permanent if it is not rapidly decompressed by surgery. The haematoma usually forms during insertion of the epidural and so most are present or developing when patients are in the immediate postoperative period. If your patient cannot move his/her legs in the recovery room - consider spinal compression. It may be that a high concentration of local anaesthetic was used during surgery which would account for the weakness. However, any motor block should

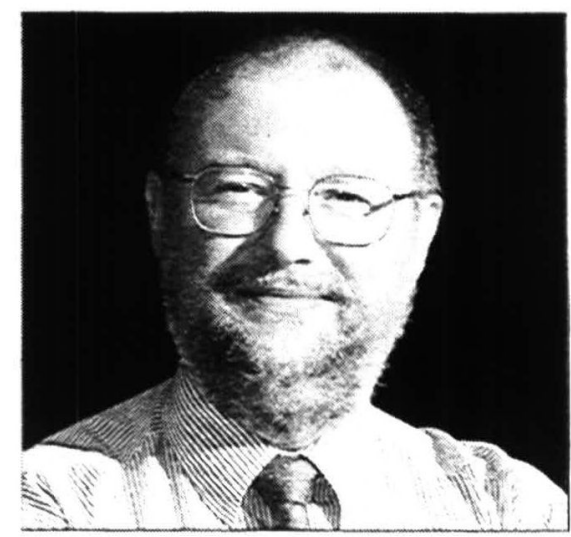

wear off rapidly after the procedure. If it even occurs to you that there may be a problem discuss it with your anaesthetic colleague. Early detection of this complication is paramount.

Epidural abscesses are rare and also cause spinal compression. Reports have quoted an incidence of 1 in 2-50 000. I believe that many of these cases go unreported and it is likely that we underestimate the problem. Aseptic technique is absolutely essential when epidurals are being inserted and this should not be compromised by any other considerations. Your expert assistance to the anaesthetist during insertion is vital.

Finally, a very significant risk in a busy recovery room is the accidental intravenous injection of local anaesthesia which has been prepared for epidural infusion. This will cause profound cardiovascular collapse and you should always be aware of the possibility of this potentially fatal error. Similarly, accidental epidural administration of some intravenous drug preparations can be disastrous. Work is underway in order to make it impossible for epidural infusions to be connected to intravenous lines (and vice versa) but you should have local practices already in place to ensure that the chances of this mistake occurring are almost zero.

Having said all this, let us remember that epidural analgesia has been a real advance in terms of patient care. However, patients require close observation with a high index of suspicion for serious complications. Members of BARNA play a significant role in ensuring patient safety and comfort in these patients.

David I Rowbotham 